\title{
Interaction of bovine serum albumin with two alkylimidazolium-based ionic liquids investigated by microcalorimetry and circular dichroism
}

\author{
Lan-Ying $\mathrm{Zhu}^{1^{*}}$, Guang-Qian $\mathrm{Li}^{2}$, Fu-Yin Zheng ${ }^{1}$ \\ ${ }^{1}$ College of Life Science and Bioengineering, Liaocheng University, Liaocheng, China; \\ *Corresponding Author: zhulanyingzly@163.com or zhulanying@1cu.edu.cn \\ ${ }^{2}$ College of Chemistry and Chemical Engineering, Liaocheng University, Liaocheng, China.
}

Received 17 January 2011; revised 2 March 2011; accepted 28 March 2011.

\begin{abstract}
The interactions of bovine serum albumin (BSA) with two alkylimidazolium-based ionic liquids, 1-butyl-3-methylimidazolium tetrafluoroborate ([bmim]BF 4 ) and 1-butyl-3-methylimidazolium hexafluorophosphate ([bmim] $\mathrm{PF}_{6}$ ), in buffer solutions at $\mathrm{pH} 7.0$ were investigated by isothermal titration calorimetry (ITC) and circular dichroism (CD). CD spectra showed that the two ionic liquids changed the secondary structure of BSA. Data process was based on the supposition that there were several independent types of binding sites on each BSA molecule for the two ligand molecules. The results obtained by using this supposition combined with Langmuir adsorption model showed that there were two types of such binding sites. One was the high affinity binding site, and the other was the low affinity binding site. The binding constants, changes in enthalpy, entropy and Gibbs free energy for the two types of binding were obtained, which showed that the two types of binding were driven by a favorable entropy increase. Furthermore, for either the ionic liquids, the number of the high affinity binding sites is much smaller than that of the low affinity ones. These results were interpreted with the molecular structure of BSA and the different substituent groups on imidazole ring of the two ionic liquid molecules.
\end{abstract}

Keywords: Isothermal Titration Calorimetry; Circular Dichroism Spectra;

Alkylimidazolium-Based Ionic Liquids; Bovine Serum Albumin

\section{INTRODUCTION}

Protein pharmaceuticals are subjected to a number of stresses during production, storage, and shipping, resulting in loss of the protein concentration and activities or formation of soluble and insoluble aggregates. The general method for stabilizing liquid protein pharmaceuticals is the use of formulation excipients. Surfactants are indispensable as solubilizing agents in the isolation and purification of proteins. To use them correctly, it is necessary to have an idea of how and in which amounts they interact with proteins. Although surfactant-protein interactions have been widely studied for half a century [1-5], the mechanism of interaction is not well understood. Knowledge of the interactions is not only fundamental in theoretics, but also practical in industrial applications. In the cosmetic and food fields, protein function is largely influenced by an added surfactant [6].

In the studies of surfactant-protein interactions, the serum albumin, e.g., human serum albumin (HSA) or bovine serum albumin (BSA), is commonly used as model protein due to its well-established primary structure, stability, water solubility and versatile binding capacity [7-9]. Ionic liquids (ILs) are a class of organic molten electrolytes at or near ambient temperature [10]. Their physical and chemical properties can be tailored by judicious selection of cation, anion, and substituent. They have no significant vapor pressures, outstanding catalytic properties, high ion-conductivity, non-flammability, and are relatively inexpensive to manufacture [11]. Thus ILs have attracted much attention as electrolytes and solvent media for reactions and extractions [12-14]. So study on binding of ionic liquid-type surfactants to bovine serum albumin would be very necessary to further understand the structural and functional information of surfactant-protein interactions. Among various ILs, the alkylimidazolium salts which belong to ionic liquid-type surfactants have been extensively studied in the field of colloid and interface science [15-17]. In this paper, 1-butyl-3-methyl imidazolium tetrafluoroborate 
([bmim $\left.] \mathrm{BF}_{4}\right)$ and 1-butyl-3-methylimidazolium hexafluorophosphate $\left([\mathrm{bmim}] \mathrm{PF}_{6}\right)$ (see Scheme 1) were selected as ligands to the protein. Isothermal titration calorimeter (ITC) was used to determine the thermodynamic parameters (enthalpy, binding site number and binding constant, etc) of the interactions of the both alkylimidazolium-based ionic liquids, [bmim $] \mathrm{BF}_{4}$ and $\left[\mathrm{bmim} \mathrm{PF}_{6}\right.$, with BSA at the temperature of $298.15 \mathrm{~K}$. CD spectroscopy was also employed to determine the dependence of $\alpha$-helical content in the protein molecules on ionic liquid concentration.

\section{MATERIALS AND METHODS}

\subsection{Materials}

Bovine serum albumin was purchased from Acros, which was used without further purification. The concentration of BSA was determined by using the extinction coefficient at $280 \mathrm{~nm}$ of $44720 \mathrm{M}^{-1} \mathrm{~cm}^{-1}$ at $\mathrm{pH} 7.0$ [18]. 1-butyl-3-methylimidazolium tetrafluoroborate ([bmim $\left.] \mathrm{BF}_{4}\right)$ and 1-butyl-3-methylimidazolium hexafluorophosphate $\left([\mathrm{bmim}] \mathrm{PF}_{6}\right)$ were both obtained from Aldrich (mass fraction $>99 \%$ ). All solutions were prepared with thrice distilled water in calorimetric experiment. Tris (hydroxymethyl) aminomethane (Tris- $\mathrm{HCl}$ ) used in the preparation of the buffer was of analytical grade.

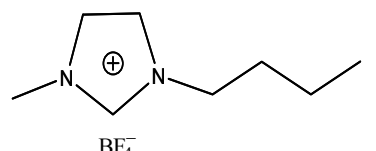

(a)

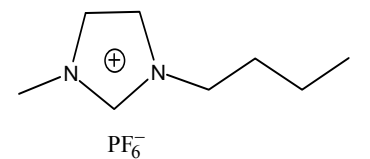

(b)
Scheme 1. Molecular structure of two ionic liquids (a) 1-butyl-3-methylimidazolium tetrafluoroborate ([bmim $\left.] \mathrm{BF}_{4}\right)$; (b) 1-butyl-3-methylimidazolium hexafluorophosphate ([bmim] $\mathrm{PF}_{6}$ ).

\subsection{Circular Dichroism (CD) Measurements}

$\mathrm{CD}$ measurements of BSA in tris- $\mathrm{HCl}$ buffer solutions of pH 7.0 were performed using a Jasco J-810 spectropolarimeter (Japan) at the temperature $298.2 \mathrm{~K}$. The light source system was protected by nitrogen (flow rate: $5 \mathrm{~L}$ $\left.\min ^{-1}\right)$. The spectra of protein solutions $(2 \mu \mathrm{M})$ were determined in $1 \mathrm{~mm}$ cells. The wavelength region of scanning was $190-250 \mathrm{~nm}$. The solutions were scanned at $100 \mathrm{~nm} \mathrm{~min} \mathrm{~m}^{-1}$ using a $1 \mathrm{~s}$ time constant with step resolution of $0.1 \mathrm{~nm}$. The average of three scans was recorded.

\subsection{Microcalorimetric Measurements}

Titration microcalorimetry was performed on a nanowatt-scale isothermal titration micorcalorimeter sup- ported by Thermal Activity Monitor TAM 2277 (Thermometric, Sweden), which was controlled by Digitam 4.1 software. This instrument has an electrical calibration with a precision better than $\pm 1 \%$ that can be determined by measuring the dilution enthalpy of a concentrated sucrose solution [19]. Each channel is a twin heat-conduction calorimeter where the heat-flow sensor is a semiconducting thermopile (multi junction thermocouple plates) positioned between the vessel holders and the surrounding heat sink. For the measurement of the protein $(\mathrm{BSA})$ - ligand $\left([\mathrm{bmim}] \mathrm{BF}_{4}\right.$ or $\left[\mathrm{bmim}^{\mathrm{b}} \mathrm{PF}_{6}\right)$ solutions, the $1 \mathrm{~mL}$ sample cell of the calorimeter made from stainless steel were initially loaded with $800 \mu \mathrm{L}$ BSA solution whose concentration was $100 \mu \mathrm{M}$. $30.00 \mathrm{mM}$ [bmim] $\mathrm{BF}_{4}$ or $[\mathrm{bmim}] \mathrm{PF}_{6}$ solution was injected into the stirred sample cell in 30 portions of $12 \mu \mathrm{L}$ using a 500 $\mu \mathrm{L}$ Hamilton syringe controlled by a Thermometric 612 Lund Pump. The interval between two injections was 35 min., which was sufficiently long for the signal to return to the baseline. The system was stirred at $30 \mathrm{rpm}$ with a gold propeller. All experiments were performed at a fixed temperature of $(298.15 \pm 0.01) \mathrm{K}$ and repeated thrice. To deduct the dilution heats of ionic liquid and BSA solutions, we performed titration experiments of ionic liquid solution into buffer solution and buffer solution into BSA solution, respectively.

\section{RESULTS AND DISCUSSION}

\subsection{Circular Dichroism Studies}

Figure 1 shows the CD spectra of BSA under the coexistence of one of the two ionic liquids at different concentrations. [bmim] $\mathrm{BF}_{4}$ and $[\mathrm{bmim}] \mathrm{PF}_{6}$ do not present any CD signal in the spectral range $190-250 \mathrm{~nm}$. This indicates that the observed CD signal is only produced by BSA. The CD spectrum of BSA exhibits two negative bands in the ultraviolet region at $208\left(\pi \rightarrow \pi^{*}\right.$ transition) and $222 \mathrm{~nm}\left(\mathrm{n} \rightarrow \pi^{*}\right.$ transition), which is characteristic of the $\alpha$-helical structure of a protein [20]. It can be seen from Figure 1 that the interaction of [bmim $] \mathrm{BF}_{4}$ or $[\mathrm{bmim}] \mathrm{PF}_{6}$ with BSA caused a slight decrease in band intensity at all wavelengths of the far UV CD without any significant shift in the peak position. This clearly indicates the minor changes in the protein secondary structure, namely the decrease in the $\alpha$-helical content in protein. This maybe caused by the interaction between the ionic liquid and BSA which leads to a swelling of the biomacromolecule and exposing of the hydrophobic residues [21]. Thus some of the original $\alpha$-helices are broken to give a more open disordered structure. However, it can be seen from Figure $\mathbf{1}$ that the change of secondary structure is quite small in 


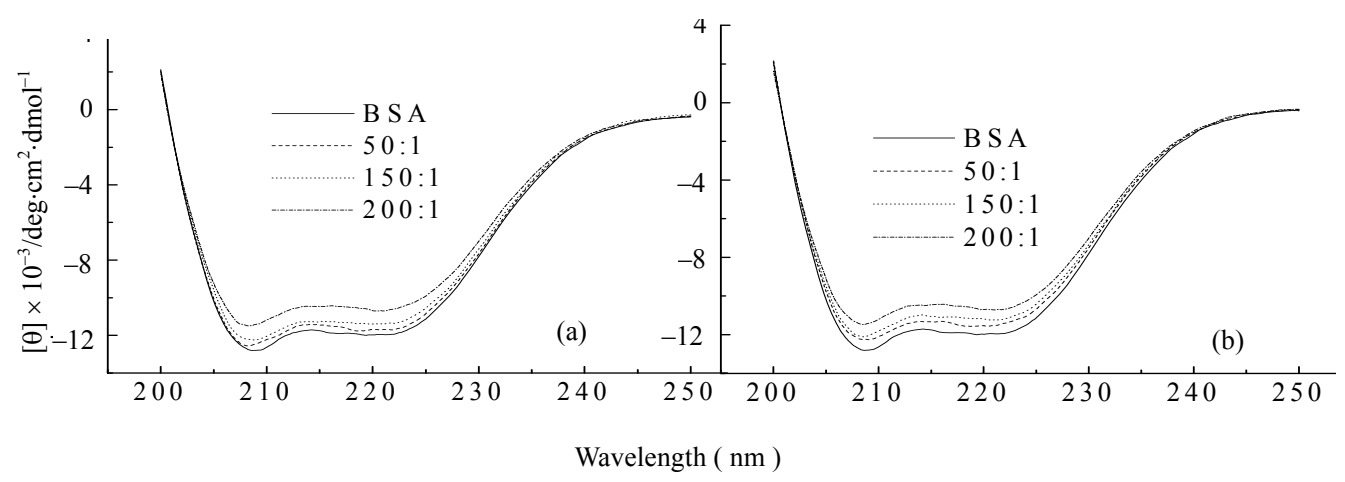

Figure 1. Circular dichroism spectra of BSA in Tris- $\mathrm{HCl}$ buffer $(10 \mathrm{mM})$ at $\mathrm{pH} 7.0$ as a function of ionic liquid concentration: (a) $\mathrm{BSA}+[\mathrm{bmim}] \mathrm{BF}_{4}$; (b) $\mathrm{BSA}+\left[\mathrm{bmim}^{-} \mathrm{PF}_{6}\right.$. (The ratio in this figure is the analytic concentration ratio of ionic liquid to BSA).

the molar ratio range of titration experiment (ionic-BSA: $135: 1)$. So we can conclude that the heat effect caused by conformational change of BSA can be neglected. That is, the measured enthalpy changes are mainly caused by the binding of the ionic liquid molecules to BSA.

\subsection{Thermodynamic Data Analysis}

\subsubsection{Titration Calorimetric Model for the Binding of Ionic Liquid to BSA}

A multiple-site model has been proposed for the binding of ligand molecules to protein, which can be regarded as adsorption of the ligand particles on the sites belonging to different classes and abiding Langmuir isotherm [4,22-23]. For the $i$-th class of binding sites, there are:

$$
\begin{gathered}
\theta_{i}=K_{i} c_{\mathrm{L}} /\left(1+K_{i} c_{\mathrm{L}}\right) \\
c_{\mathrm{L}, 0}=c_{\mathrm{L}}+c_{\mathrm{P}, 0} \sum_{i=1}^{m} N_{i} \theta_{i}
\end{gathered}
$$

where $\theta_{i}$ is the degree of occupancy of the $i$-th types of sites. $c_{\mathrm{L}, 0}$ and $c_{\mathrm{P}, 0}$ indicate total concentration of ligand and protein, respectively. $c_{\mathrm{L}}$ is concentration of free ligand molecule at equilibrium state and $m$ is the number of binding classes.

The net heat of interaction between the ligand molecule and the biomacromolecule evolved from the $j$-th injection in an ITC experiment can be expressed

$$
Q_{j}=c_{\mathrm{P}, 0} V_{\mathrm{P}, 0} \sum_{i=1}^{m} N_{i} \Delta \theta_{i} \Delta H_{i}^{\mathrm{o}}
$$

where $V_{\mathrm{P}, 0}$ is the volume of the protein solution as titrand in the calorimeter cell and $\Delta \theta_{i}$ is the change in occupancy from the $(j-1)$ th injection to the $j$ th one.

Eq.1 and Eq. 2 indicate that $c_{\mathrm{L}}$ is the function of $N_{i}$ and $K_{i}$ when $c_{\mathrm{L}, 0}$ and $c_{\mathrm{P}, 0}$ are known. So there are $3 \mathrm{~m}$ unknown parameters in (3), which are $K_{i}, \Delta H_{i}^{\mathrm{o}}$ and $N_{i}$.
These parameters for single-class ( $m=1)$, two-class ( $m$ $=2)$ and three-class $(m=3)$ binding model were computed from the actual calorimetric data with an iterative non-linear least-square regression program for minimizing the value of $\Sigma\left(Q_{\text {exp }}-Q_{\text {calc }}\right)^{2}$ by using of software MATLAB 7.01. The coincidence degree between calculated curve and experimental integrate heat indicates that existence of two types of binding sites is most reasonable when either $[\mathrm{bmim}] \mathrm{BF}_{4}$ or $[\mathrm{bmim}] \mathrm{PF}_{6}$ binds to BSA. The nonlinear fitting curves of integrated heat versus the mixed ligand/BSA molar ratio are shown in Figure 2. According to the thermodynamic formula:

$$
\Delta G^{\mathrm{o}}=-R T \ln K^{\mathrm{o}} \Delta G^{\mathrm{o}}=\Delta H^{\mathrm{o}}-T \Delta S^{\mathrm{o}}
$$

Standard changes of Gibbs free energy $\left(\Delta G^{\circ}\right)$ and entropy effect $\left(T \Delta S^{\circ}\right)$ for the binding process of ionic liquid to BSA can be derived. The thermodynamic results for the two ligand molecules + BSA complexes are listed in Table 1.

\subsubsection{Binding Site Number and Binding Constants}

Nonlinear fitting curves of integrated heat versus the mixed ionic liquid/BSA molar ratio are shown in Figure 2. It can be seen that the binding of the two ionic liquids to BSA is exothermic in the range of selected ionic liquid concentration. Crystal structure analyses have revealed that the binding sites on BSA molecules are located in subdomains IIA and IIIA. A large hydrophobic cavity is present in the IIA subdomain [24]. Moreover, due to the presence of amino acid residues on the surface of HSA, most authors agree on the existence of a limited number of binding sites on the surface of protein molecules [25]. The optimum simulated results show that there are two types of binding sites on BSA molecules for the binding of the two ionic liquids to BSA: 1) binding to ionic sites on BSA surface driven by hydrogen bonding interaction of anions of $\left[\mathrm{bmim}^{\mathrm{B}}\right] \mathrm{BF}_{4}$ or 


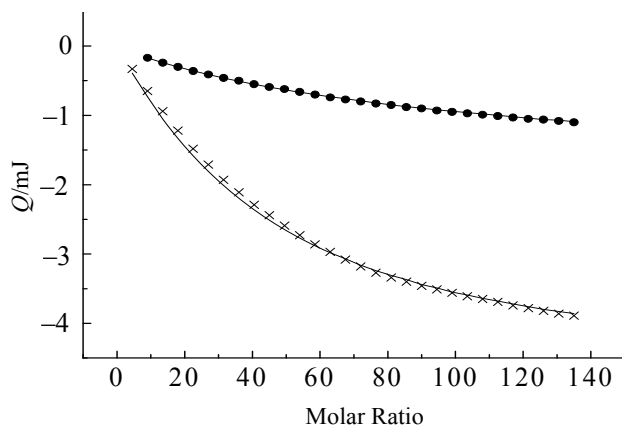

Figure 2. Non-linear fitting curve of the binding heat versus the molar ratio of the two ionic liquid to BSA, where points are gotten from experiments and the solid line is the result of simulation, $\times$ : $\left[\mathrm{bmim}^{\mathrm{B}} \mathrm{BF}_{4}\right.$; $\bullet$ : $[\mathrm{bmim}] \mathrm{PF}_{6}$.

[bmim $] \mathrm{PF}_{6}$ with amino acid residues on BSA surface, which corresponds to the high affinity sites; 2) hydrophobic interaction of imidazole ring and the hydrophobic chain on either of the two ionic liquids with the hydrophobic cavities of BSA molecules, which corresponds to the low affinity sites. The work of Swati De et al. indicates that high affinity sites corresponding to the ionic sites on protein surface, and low affinity sites corresponding to the hydrophobic cavities of protein molecules [26]. From the data in Table 1, we can reach two conclusions, which are: when the same ionic liquid binds to BSA, the high affinity site number $\left(N_{1}\right)$ is smaller than the low affinity site number $\left(N_{2}\right)$, while the binding constant for the high affinity sites is evidently larger than that for the low affinity sites. This difference may be explained as follows. The isoelectric point of serum album is at $\mathrm{pH} 4.70$ [27] and BSA molecule is negatively charged at $\mathrm{pH}$ 7.0. The actually ligand particles are [bmim $]^{+}$cations, for which the mainly binding force to the first type of sites is electrostatic interaction. This can also explain the fact that the two values of the equilibrium constant $K_{1}$ respectively for $[\mathrm{bmim}] \mathrm{BF}_{4}$ and [bmim $] \mathrm{PF}_{6}$ are almost the same. As to the difference between the two values of $N_{1}$ respectively corresponding to $[\mathrm{bmim}] \mathrm{BF}_{4}$ and $[\mathrm{bmim}] \mathrm{PF}_{6}$ might be interpreted from the structures of the two anions, $\mathrm{BF}_{4}^{-}$and $\mathrm{PF}_{6}^{-}$. The size of the former is smaller than the later, and so there is more opportunity for the former to approach the biomacromolecule, which obstructs the ligand cation i.e. decrease the binding site. The relative differences between values of $K_{2}, N_{2}, \Delta H_{2}^{\circ}, T \Delta S_{2}^{\circ}$ respectively corresponding to $[\mathrm{bmim}] \mathrm{BF}_{4}$ are all not evident. This phenomenon indicate that the actually ligand is also the cation in the second type of binding process driven by hydrophobic interaction.

\subsubsection{Enthalpy and Entropy Effects}

The standard enthalpy effects, standard entropy effects
Table 1. Thermodynamic parameters for the binding of [bmim] $\mathrm{BF}_{4}$ and $\left[\mathrm{bmim}^{\mathrm{b}} \mathrm{PF}_{6}\right.$ to $\mathrm{BSA}$ at $298.15 \mathrm{~K}^{a}$.

\begin{tabular}{ccc}
\hline \multirow{2}{*}{ Parameter } & \multicolumn{2}{c}{ ionic liquid } \\
\cline { 2 - 3 } & {$[\mathrm{bmim}] \mathrm{BF}_{4}$} & {$[\mathrm{bmim}] \mathrm{PF}_{6}$} \\
\hline High affinity sites & $1.0 \pm 0.2$ & $3.1 \pm 0.1$ \\
$N_{1}$ & $(1.12 \pm 0.04) \times 10^{3}$ & $(1.48 \pm 0.07) \times 10^{3}$ \\
$K_{1} /\left(\mathrm{M}^{-1}\right)$ & $-2.14 \pm 0.08$ & $-2.46 \pm 0.09$ \\
$\Delta H_{1} /\left(\mathrm{kJ} \cdot \mathrm{mol}^{-1}\right)$ & $-17.40 \pm 0.19$ & $-18.09 \pm 0.28$ \\
$\left.\Delta G_{1}{ }^{\circ} / \mathrm{kJ} \cdot \mathrm{mol}^{-1}\right)$ & $15.26 \pm 0.27$ & $15.63 \pm 0.37$ \\
$T \Delta S_{1}{ }^{\circ} /\left(\mathrm{kJ} \cdot \mathrm{mol}^{-1}\right)$ & & \\
$\mathrm{Low} \mathrm{affinity} \mathrm{sites}$ & $25.0 \pm 0.9$ & $27.0 \pm 1.0$ \\
$N_{2}$ & $65.20 \pm 2.60$ & $98.50 \pm 3.50$ \\
$K_{2} /\left(\mathrm{M}^{-1}\right)$ & $-1.38 \pm 0.05$ & $-1.52 \pm 0.06$ \\
$\Delta H_{2}{ }^{\circ} /\left(\mathrm{kJ} \cdot \mathrm{mol}^{-1}\right)$ & $-10.36 \pm 0.12$ & $-11.38 \pm 0.18$ \\
$\Delta G_{2}{ }^{\circ} /\left(\mathrm{kJ} \cdot \mathrm{mol}^{-1}\right)$ & $8.98 \pm 0.17$ & $9.86 \pm 0.24$ \\
$T \Delta S_{2}{ }^{\mathrm{o}} /\left(\mathrm{kJ} \cdot \mathrm{mol}^{-1}\right)$ &
\end{tabular}

${ }^{a}$ Data are expressed as mean \pm S. D. $(N=3)$.

and changes in standard Gibbs free energies for the binding of $[\mathrm{bmim}] \mathrm{BF}_{4}$ and $\left[\mathrm{bmim}^{\mathrm{b}} \mathrm{PF}_{6}\right.$ to $\mathrm{BSA}$ are shown in Figure 3. It is necessary to know the main existing form of the two ionic liquids in sample cell in order to interpret the above experimental results. The critical micelle concentrations $(\mathrm{CMC})$ of $\mathrm{BF}_{4}$ and $[\mathrm{bmim}] \mathrm{PF}_{6}$ are about $0.8 \mathrm{M}$ [28] and $0.031 \mathrm{M}$ [29] respectively, which exceed the initial concentration $(30.00 \mathrm{mM})$ of the two ionic liquids in the syringe. Therefore, the two ionic liquids in the sample cell are both existent as monomers instead of micelles. In other words, there is no demicellization of the ionic-liquid-type surfactant in the experimental concentration ranges. Furthermore, as described above, the heat effect caused by conformational change of BSA can be neglected. It can be seen from Figure 3 that the standard enthalpies of formation for the binding of the two ionic liquids to the both types of binding sites of BSA are negative, which indicates that the binding process are both exothermic. The reasons for the negative values of $\Delta H_{1}^{0}$ are manifold. Firstly, the electrostatic attraction of the cation, $[\mathrm{bmim}]^{+}$, with the negatively charged protein molecule is an exothermic process. Secondly, the repulsion force between the anions of the ionic liquid and the negatively charged BSA surface makes positive contribution to $\Delta H_{1}^{\mathrm{o}}$. Thirdly, destroy of iceberg structure surrounding hydrophobic chains of the ionic liquid is an endothermic process. The negative values of $\Delta H_{1}^{0}$ indicate that the hydrogen bonding interaction is predominant over the electrostatic repulsion interaction and the destroy effects of iceberg structure. As for $\Delta H_{2}^{\circ}$, there are also several types of weak interactions simultaneously contribute to its negative value, including the expulsion of high energy water molecule from hydrophobic cavity of the protein molecule to bulk solution (exothermic process), destroy of iceberg structure surrounding hydrophobic groups of the ionic liquid 


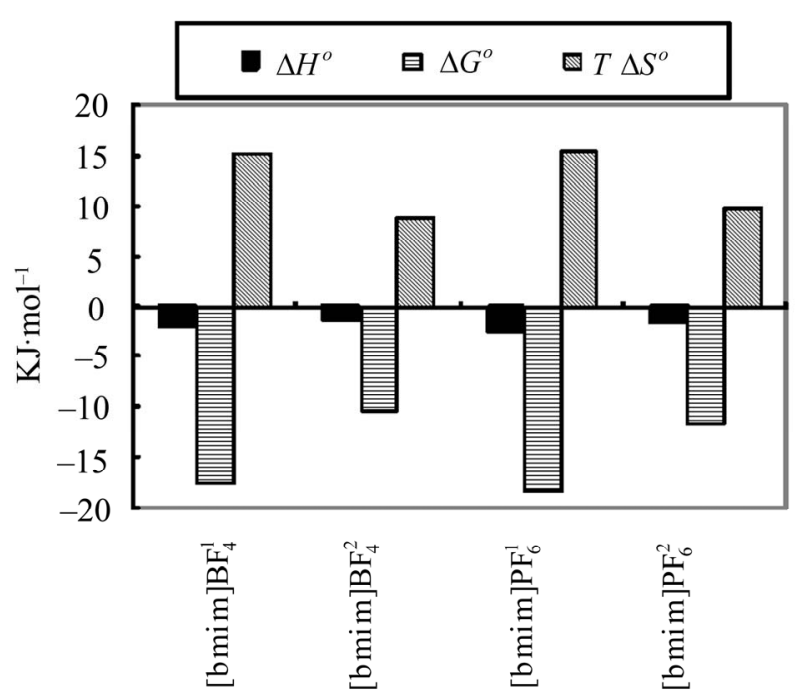

Figure 3. Proportion of the thermodynamic parameters for the binding of bmim] $\mathrm{BF}_{4}$ and [bmim] $\mathrm{PF}_{6}$ to BSA (Superscript 1 and 2 represent high affinity sites and low affinity sites, respectively.).

(endothermic process), and the hydrophobic interaction of imidazole ring and the hydrophobic chain on it with the hydrophobic cavities of BSA molecules (exothermic process) [30]. Among them, the hydrophobic interaction should be the main driving force, evidenced by the experimental results that the difference between the $\Delta H_{2}^{\circ}$ for $[\mathrm{bmim}] \mathrm{BF}_{4}-\mathrm{BSA}\left(-1.38 \pm 0.05 \mathrm{~kJ} \cdot \mathrm{mol}^{-1}\right)$ and that for $\left[\mathrm{bmim} \mathrm{PF}_{6}-\mathrm{BSA}\left(-1.52 \pm 0.06 \mathrm{~kJ} \cdot \mathrm{mol}^{-1}\right)\right.$ is subtle because the two ionic liquids contain the same hydrophobic chain.

It can be seen from Figure 3 that the entropy effects for the binding of [bmim] $\mathrm{BF}_{4}$ and $\left[\mathrm{bmim}^{\mathrm{b}} \mathrm{PF}_{6}\right.$ to $\mathrm{BSA}$ are all positive, which is beneficial for this interacting process. This may be due to the total result of the binding of the two ligand ions to BSA molecule (negative contribution to entropy) and the releasing of water molecules from cavity (positive contribution to entropy) as well as the disruption of hydration layer structure on the surface of BSA molecule (positive contribution to entropy). In addition, we can infer from Figure 3 that the binding process is predominantly entropy driven.

\section{CONCLUSIONS}

ITC experiments demonstrate that there are two types of binding sites on BSA molecules for the Alkylimidazolium cation in the both ionic liquids, $[\mathrm{bmim}] \mathrm{BF}_{4}$ and [bmim $] \mathrm{PF}_{6}$. One type of binding with high affinity binding is caused electrostatic interaction of the cation with negatively charged sites on BSA molecules, and the other one is low affinity binding due to the hydrophobic interaction of imidazole ring and the hydrophobic chain on it of the both ionic liquids with the hydrophobic cavities of the protein molecules. The thermodynamic results obtained from the calorimetric data with an iterative non-linear least-square regression program show that when the same ionic liquid cations bind to BSA molecules, the number of high affinity sites $\left(N_{1}\right)$ is smaller than that of the low affinity sites $\left(N_{2}\right)$. On the contrary, the binding constant for the high affinity sites $\left(K_{1}\right)$ is evidently larger than that for the low affinity sites $\left(K_{2}\right)$. The entropy effects for the two binding sites are both negative while the entropy effects for the two binding sites are both positive. Circular dichroism (CD) spectra show that the two ionic liquids change the secondary structure of BSA. These results can be understood by considering several weak interactions of the biomacromolecule with the cation, the anion as well as the solvent effect of water on the protein-ligand interaction system. The data and message obtained in this study may be important for understanding the influence of protein-surfactant interactions on the functionality of globular proteins in the biochemical systems.

\section{ACKNOWLEDGEMENTS}

The authors are grateful to Inovation Project of Shandong Province, LCUYZ10008.

\section{REFERENCES}

[1] Kelley, D. and McClements, D.J. (2003) Interactions of bovine serum albumin with ionic surfactants in aqueous solutions. Food Hydrocolloids, 17, 73-85. doi:10.1016/S0268-005X(02)00040-1

[2] Ruiz-Peña, M., Oropesa-Nuñez, R., Pons, T., Louro, S.R.W. and Pérez-Gramatgesa, A. (2010) Physicochemical studies of molecular interactions between non-ionic surfactants and bovine serum albumin. Colloids and Surfaces B: Biointerfaces, 75, 282-289. doi:10.1016/j.colsurfb.2009.08.046

[3] Valstar, A., Almgren, M. and Brown, W. (2000) The interaction of bovine serum albumin with surfactants studied by light scattering. Langmuir, 16, 922-927. doi:10.1021/la990423i

[4] Liu, M., Sun, D. Z., Li, N. and Zhu, L.Y. (2009) Studies on the binding of bis-quaternary ammonium surfactants to bovine serum albumins by microcalorimetry and circular dichroism. Journal of Dispersion Science and Technology, 30, 459-465. doi:10.1080/01932690802548635

[5] Gelamo, E.L., Itri, R., Alonso, A., Silva, J.V. and Tabak, M. (2004) Small-angle X-ray scattering and electron paramagnetic resonance study of the interaction of bovine serum albumin with ionic surfactants. Journal of Colloid and Interface Science, 277, 471-482. doi:10.1016/j.jcis.2004.04.065

[6] Geng, F., Zheng, L.Q., Yu, L., Li, G.Z. and Tung, C.H. (2010) Interaction of bovine serum albumin and long-chain imidazolium ionic liquid measured by fluo- 
rescence spectra and surface tension. Process biochemistry, 45, 306-311. doi:10.1016/j.procbio.2009.10.001

[7] Trynda-Lemiesz, L. (2004) Paclitaxel-HSA interaction. Binding sites on HSA molecule. Bioorganic \& Medicinal Chemistry, 12, 3269-3275. doi:10.1016/i.bmc.2004.03.073

[8] Barbosa, S., Taboada, P., Attwood, D. and Mosquera, V. (2003) Thermodynamic properties of the complex formed by interaction of two anionic amphiphilic penicillins with human serum albumin. Langmuir, 19, 10200-10204. doi:10.1021/la035106x

[9] $\mathrm{Hu}$, Y.J., Liu, Y., Pi, Z.B. and Qu, S.S. (2005) Interaction of cromolyn sodium with human serum albumin: A fluorescence quenching study. Bioorganic \& Medicinal Chemistry, 13, 6609-6616. doi:10.1016/j.bmc.2005.07.039

[10] Li, R.X. (2004) Green solvent: Synthesis and application of ionic liquids. Chemistry Technology Press, Beijing.

[11] Robin, D.R. and Kenneth, R.S. (2003) Ionic liquids - Solvents of the future. Science, 302, 792-793.

[12] McEwen, A.B., Ngo, H.L., Lecompte, K. and Goldman, J.L. (1999) Electrochemical properties of imidazolium salt electrolytes for electrochemical capacitor applications. Journal of the Electrochemical Society, 146, 1687-1695. doi:10.1149/1.1391827

[13] Welton, T. (1999) Room-temperature ionic liquids. Solvents for synthesis and catalysis. Chemical Review, 99, 2071-2084. doi:10.1021/jp020631a

[14] Anthony, J.L., Maginn, E.J. and Brennecke, J.F. (2002) Solubilities and thermodynamic properties of gases in the ionic liquid 1- $n$-butyl-3-methylimidazolium hexafluorophosphate. The Journal of Physical Chemistry B, 106, 7315-7320. doi:10.1021/jp020631a

[15] He, Y.F. and Shen, X.H. (2008) Interaction between $\beta$-cyclodextrin and ionic liquids in aqueous solutions investigated by a competitive method using a substituted $3 \mathrm{H}$-indole probe. Journal of Photochemistry and Photobiology A: Chemistry, 197, 253-259. doi:10.1016/j.jphotochem.2008.01.001

[16] Archer, D. G., Widegren, J. A., Kirklin, D. R. and Magee, J. W. (2005) Enthalpy of solution of 1-octyl-3-methylimidazolium tetrafluoroborate in water and in aqueous sodium fluoride. Journal of Chemical \& Engineering Data, 50, 1484-1491. doi:10.1021/je050136i

[17] Chen, Y.H., Meng, Y.S., Zhang, S.M., Zhang, Y., Liu, X.W. and Yang, J. (2010) Liquid-liquid equilibria of aqueous biphasic systems composed of 1-bButyl3-methyl imidazolium tetrafluoroborate + sucrose/maltose + water. Journal of Chemical \& Engineering Data, 55, 3612-3616. doi:10.1021/je100212p

[18] Moriyama, Y. and Takeda, K. (2005) Protective effects of small amounts of bis(2-ethylhexyl) sulfosuccinate on the helical structures of human and bovine serum albumins in their thermal denaturations. Langmuir, 21, 5524-5528. doi:10.1021/1a050252j

[19] Bai, G., Wang, Y. and Yan, H. (2002) Thermodynamics of interaction between cationic gemini surfactants and hydrophobically modified polymers in aqueous solutions. The Journal of Physical Chemistry B, 106, 2153-2159. doi:10.1021/jp0123839

[20] Roy, A.S., Tripathy, D.R., Chatterjee, A. and Dasgupta, S. (2010) A spectroscopic study of the interaction of the antioxidant naringin with bovine serum albumin. Journal of Biophysical Chemistry, 1, 141-152. doi:10.4236/jbpc.2010.13017

[21] Nielsen, A. D., Borch, K. and Westh, P. (2000) Thermochemistry of the specific binding of C12 surfactants to bovine serum albumin. Biochimica et Biophysica Acta, 1479, 321-331. doi:10.1016/S0167-4838(00)00012-1

[22] Aberkane, L., Jasniewski, J., Gaiani, C., Scher, J. and Sanchez, C. (2010) Thermodynamic characterization of acacia gum- $\beta$-lactoglobulin complex coacervation. Langmuir, 26, 12523-12533. doi:10.1021/la100705d

[23] Liu, M., Zhu, L.Y., Qu, X.K., Sun, D.Z. and Li, L.W. (2007) Studies on the binding of paeonol and two of its isomers to human serum albumin by using microcalorimetry and circular dichroism. The Journal of Chemical Thermodynamics, 39, 1565-1570. doi:10.1016/j.jct.2007.05.003

[24] Sulkowska, A. (2002) Interaction of drugs with bovine and human serum albumin. Journal of Molecular Structure, 614, 227-232. doi:10.1016/S0022-2860(02)00256-9

[25] Gelamo, E.L. Tabak, M. (2000) Spectroscopic studies on the interaction of bovine (BSA) and human (HSA) serum albumins with ionic surfactants. Spectrochimica Acta Part A: Molecular and Biomolecular Spectroscopy, 56, 2255-2271. doi:10.1016/S1386-1425(00)00313-9

[26] De, S., Ginishwar, A. and Das, S. (2005) Fluorescence probing of albumin-surfactant interaction. Journal of Colloid and Interface Science, 285, 562-573. doi:10.1016/j.jcis.2004.12.022

[27] Liu, R.T., Yang, J.H., Sun, C.X., Wu, X., Li, L. and Li Z. (2003) Resonance light-scattering method for the determination of BSA and HSA with sodium dodecyl benzene sulfonate or sodium lauryl sulfate. Analytical and Bioanalytical Chemistry, 377, 375-379. doi:10.1007/s00216-003-2091-y

[28] Dong, B., Zhao, X. Y., Zheng, L. Q., Zhang, J., Li, N. and Inoue, T. (2008) Aggregation behavior of long-chain imidazolium ionic liquids in aqueous solution: Micellization and characterization of micelle microenvironment. Colloids and Surfaces A: Physicochemical and Engineering Aspects, 317, 666-672. doi:10.1016/j.colsurfa.2007.12.001

[29] Deng, X. (2008) Study on ionic liquids used as solvent for fluorescence analysis. MA dissertation, Southwest University, Chongqing.

[30] Sun, D.Z., Li, L., Qiu, X.M., Liu, F. and Yin, B.L. (2006) Isothermal titration calorimetry and ${ }^{1} \mathrm{H}$ NMR studies on host-guest interaction of paeonol and two of its isomers with $\beta$-cyclodextrinInt. International Journal of Pharmaceutics, 316, 7-13. doi:10.1016/j.ijpharm.2006.02.020 